\title{
Opioid Modulation of Scratching and Spinal c-fos Expression Evoked by Intradermal Serotonin
}

\author{
Hiroshi Nojima, ${ }^{1}$ Christopher T. Simons, ${ }^{2}$ Jason M. Cuellar, ${ }^{2}$ Mirela Iodi Carstens, ${ }^{2}$ Justin A. Moore, ${ }^{2}$ and Earl Carstens ${ }^{2}$ \\ ${ }^{1}$ Department of Applied Pharmacology, Faculty of Pharmaceutical Sciences, Toyama Medical and Pharmaceutical University, Toyama 930-0194, Japan, and \\ ${ }^{2}$ Section of Neurobiology, Physiology, and Behavior, University of California, Davis, Davis, California 95616
}

\begin{abstract}
We investigated a spinal site for opioid modulation of itch-related scratching behavior in rats. Intradermal 5-HT (2\%, $10 \mu \mathrm{l})$ elicited intermittent bouts of hindlimb scratching directed toward the injection site (nape of neck) beginning within minutes and lasting $>1$ hr. 5-HT-evoked scratching was significantly reduced by systemic administration of the opiate antagonist naltrexone but was not affected by systemic morphine at a dosage $(3 \mathrm{mg} / \mathrm{kg}$ ) that induces analgesia. Intradermal 5-HT elicited a significant increase in c-fos-like immunoreactivity (FLI) in superficial laminas I-III at the lateral aspect of the cervical C3-C6 dorsal horn compared with controls receiving intradermal saline. Neither systemic morphine nor naltrexone significantly affected counts of 5-HT-evoked FLI. The lack of effect of morphine suggests that intradermal 5-HT activates dorsal horn neurons, signaling itch but not pain. Attenuation of 5-HT-evoked scratching but not spinal FLI by naltrexone suggests a supraspinal site for its antipruritic action. In contrast, morphine significantly attenuated FLI elicited by intradermal capsaicin, a chemical that induces pain but not scratching.
\end{abstract}

Key words: itch; serotonin; scratching; c-fos immunoreactivity; naltrexone; morphine; rat

\section{Introduction}

Itch is an unpleasant sensation associated with the desire to scratch. Although some types of commonplace itch can be relieved by antihistamines, chronic itch associated with pathological skin conditions or systemic liver or kidney disease is often resistant to antihistamines or other pharmacologic treatments (Wahlgren, 1991; Jones and Bergasa, 2000). An increasing effort is being made to understand itch mechanisms and mediators in the hopes of improving therapeutic treatments.

Two important recent developments have prompted a renewed interest in the study of itch mechanisms. One is the development of animal itch models based on the close association of itch with scratching behavior. Scratching is elicited in rodents by pruritogenic agents (e.g., 5-HT) but not by pain-producing agents such as capsaicin or formalin (Kuraishi et al., 1995; Woodward et al., 1995; Thomsen et al., 2001; Jinks and Carstens, 2002). Interestingly, histamine, an itch inducer in humans, evokes little scratching in Sprague Dawley rats and certain mouse strains (Kitagawa et al., 1997; Maekawa et al., 2000; Inagaki et al., 2001; Thomsen et al., 2001; Jinks and Carstens, 2002). A second development is the discovery of potential itch-selective sensory receptors with slowly conducting unmyelinated fibers that are mechanically insensitive yet respond to histamine over a time course matching that of itch sensation (Schmelz et al., 1997, 2003), as well as second-order spinothalamic tract neurons having similar

\footnotetext{
Received July 29, 2003; revised Sept. 3, 2003; accepted Sept. 12, 2003.

This work was supported by California Tobacco-Related Disease Research Program Grants 6RT-0231 and 11RT0053 and National Institutes of Health Grants NS 35788 and DE 13685.

Correspondence should be addressed to E. Carstens, Section of Neurobiology, Physiology, and Behavior, University of California, Davis, 1 Shields Avenue, Davis, CA 95616. E-mail: eecarstens@ucdavis.edu.

Copyright $\odot 2003$ Society for Neuroscience $\quad$ 0270-6474/03/2310784-07\$15.00/0
}

properties (Andrew and Craig, 2001). These studies support the long-held belief that itch may be signaled by a specific sensory pathway distinct from that conveying pain, as suggested by the observations that noxious counterstimuli inhibit itch (Ward et al., 1996), that opiate analgesics can elicit itch (Lanz et al., 1982; Stenseth et al., 1985; Ballantyne et al., 1988), and that intraneural or cutaneous electrical stimulation can occasionally elicit a sensation of itch that does not become painful at higher stimulus frequencies (Tuckett, 1982; Schmidt et al., 1993).

In the present study, we used intradermal 5-HT as a presumed itch mediator to investigate opiate modulation of scratching behavior and activation of potential itch-signaling spinal neurons in rats. In ddY mice, intradermal 5-HT elicits scratching in a dose-related manner, with reduced scratching at very high doses possibly attributable to pain (Yamaguchi et al., 1999). We reasoned that 5-HT primarily elicits itch at submaximal doses. Furthermore, given that opiates sometimes elicit itch in humans and that opiate antagonists reduce experimental itch (Heyer et al., 1997), we tested whether 5-HT-evoked scratching was similarly affected by morphine and by the opiate antagonist naltrexone. We also investigated underlying neural correlates using the method of c-fos immunohistochemistry. Fos-like immunoreactivity (FLI) is elicited in dorsal horn neurons by a variety of noxious stimuli, including pruritogens (Presley et al., 1990; Yao et al., 1992; Doi-Saika et al., 1997; Luo et al., 1998; Jinks et al., 2002). In the present study, we used FLI as a marker of neuronal activation elicited by intradermal 5-HT and investigated whether FLI was affected by naltrexone and morphine in a manner comparable with their effects on 5-HT-evoked scratching behavior. For comparison, the effect of morphine on FLI elicited by the algesic chemical capsaicin was also investigated. 


\section{Materials and Methods}

Animals. Adult male Sprague Dawley rats (Simonsen Laboratories, Gilroy, CA) weighing 560-760 gm were used. The experimental protocol was approved by the University of California, Davis Animal Use and Care Advisory Committee. Rats were housed in a room under controlled temperature $\left(22 \pm 1^{\circ} \mathrm{C}\right)$ and light (lights on from 8:00 A.M. to 8:00 P.M.). Food and water were available ad libitum.

Drugs. Serotonin (5-HT) hydrochloride (Sigma, St. Louis, MO), naltrexone hydrochloride (DuPont, Garden, NY), and morphine sulfate (Mallinckrodt, St. Louis, MO) were dissolved in sterile physiological saline $(0.9 \% \mathrm{NaCl})$. The $5-\mathrm{HT}$ was prepared fresh daily at a concentration of $2 \%$ (94 mm). Capsaicin (Sigma) was diluted with sterile distilled water to a final concentration of $0.03 \%$ ( $1 \mathrm{~mm}$ ) from a stock solution of 0.1 in $40 \%$.

Intradermal microinjections and observation of scratching behavior. At least $3 \mathrm{~d}$ before receiving intradermal 5-HT, fur at the nape of each rat's neck was carefully clipped. Procedures for intradermal injections and observation of scratching behavior were as described previously (Jinks and Carstens, 2002). Briefly, all animals were habituated to the observation chamber $(30 \times 30 \times 40 \mathrm{~cm})$ in three daily sessions before formal testing and on test days for 30 min before injections. For intradermal injections, each rat was placed in a loose Plexiglas restrainer (to which it had also been habituated on three separate days before testing) that had an opening above the rat's neck. Skin at the nape of the neck was gently grasped with forceps, and the injection needle (30 gauge) was quickly inserted intradermally at the midline. 5-HT (or saline vehicle) was injected in a volume of $10 \mu \mathrm{l}$ using a $50 \mu \mathrm{l}$ Hamilton microsyringe through polyethylene-50 tubing connected to the needle. The needle was left in place for $10 \mathrm{sec}$ after injection, removed, and the rat was then quickly put back into the observation chamber and videotaped from above using a digital video camcorder (12-bit Optura Pi; Canon, Irvine, CA) for 60 $\mathrm{min}$. Three or four rats were injected and videotaped simultaneously in separate chambers. Experimenters were not in the room during videotaping. Videotapes were subsequently reviewed to score scratching behavior. A series of one or more scratching movements by the hindpaw that was directed toward the injection site was defined as a scratching bout, which ended when the rat either licked or bit the hindpaw or placed it back down on the floor. The duration of each individual scratching bout was timed with a stopwatch. The total number of scratching bouts was counted at $2 \mathrm{~min}$ intervals throughout the $60 \mathrm{~min}$ observation period to determine the time course of scratching (see Fig. 1). The degree of scratching was quantified as the total number of bouts as well as the cumulative duration of bouts (summed at 2 min intervals) over the 60 min observation period. Both measures were recently validated as indicators of scratching magnitude in rats (Nojima and Carstens, 2003).

Rats were assigned to one of four treatment groups. The first group $(n=8)$ served as control, with each rat being tested in two separate sessions at least $4 \mathrm{~d}$ apart. In the first session, rats received intradermal saline as a control for scratching behavior. In the second session, rats received intradermal 5 -HT preceded by intraperitoneal saline as a vehicle treatment control. The second group of rats $(n=8)$ received morphine $(3 \mathrm{mg} / \mathrm{kg}$, i.p.) $30 \mathrm{~min}$ before intradermal saline. In the third group $(n=$ 8 ), rats received intradermal 5 -HT preceded by intraperitoneal saline (vehicle control) in the first session and intradermal 5-HT preceded by naltrexone ( $5 \mathrm{mg} / \mathrm{kg}$, i.p.) in the second session. In the fourth group $(n=$ 6 ), rats received intradermal 5-HT preceded by intraperitoneal saline in the first session and intradermal 5-HT preceded by morphine $(3 \mathrm{mg} / \mathrm{kg}$, i.p.) in the second. Within- and between-group differences in the number of scratching bouts were assessed by ANOVA with post hoc least significant difference (LSD) tests. A $p$ value of $<0.05$ was considered significant.

$c$-fos immunohistochemistry. At least 2 weeks after the completion of behavioral testing, the rats were used in a terminal c-fos experiment. After induction of anesthesia with sodium pentobarbital (65 mg/kg, i.p.), animals received one of the following treatments: The first (control) group ( $n=5$ rats) received a $10 \mu \mathrm{l}$ intradermal injection of saline. All intradermal injections were made into the skin at the nape of the neck, $5 \mathrm{~mm}$ lateral to the midline. The injection region corresponds to the $\mathrm{C} 2-\mathrm{C} 6$ dermatomes (Takahashi and Nakajima, 1996). The second group $(n=6)$ received morphine $(3 \mathrm{mg} / \mathrm{kg}$, i.p. $) \sim 30 \mathrm{~min}$ before intradermal injection of saline. The morphine was administered $15 \mathrm{~min}$ before the induction of anesthesia and thus $\sim 30 \mathrm{~min}$ before the intradermal saline injection. The third group $(n=7)$ received intradermal 5-HT $(2 \%) \sim 15$ min after induction of anesthesia. The fourth group $(n=6)$ received naltrexone (5 $\mathrm{mg} / \mathrm{kg}$, i.p.) followed $\sim 30 \mathrm{~min}$ later by intradermal 5 -HT using the same timing of intraperitoneal and intradermal injections as in the second group. The fifth group $(n=6)$ received morphine $(3 \mathrm{mg} / \mathrm{kg}$, i.p. $) \sim 30$ min before intradermal 5-HT using the same timing sequence as above. The sixth group $(n=5)$ received intradermal injection of capsaicin $(0.03 \%)$. The seventh group $(n=6)$ received morphine $(3 \mathrm{mg} / \mathrm{kg}$, i.p.) followed $\sim 30$ min later by intradermal capsaicin. All animals were observed continually during and after intradermal injections, and none exhibited any hindlimb movements directed toward the neck. Thus, these experiments assessed the sensory but not the motor consequences of the intradermal injections.

Two hours after intradermal injections, each animal was perfused through the aorta with $250 \mathrm{ml}$ of isotonic PBS followed by $500 \mathrm{ml}$ of $4 \%$ paraformaldehyde (Jinks et al., 2002). The cervical spinal cord was then removed, postfixed for $8 \mathrm{hr}$, and transferred to a $30 \%$ sucrose solution. Two to three days later, 18 of the cervical spinal cords were cut in $50 \mu \mathrm{m}$ frozen coronal sections at C1-C6 levels and collected in three separate 24 well containers (Costar, Cambridge, MA) containing PBS. One cervical cord was cut in horizontal sections (see Fig. 2). One container was then processed immunohistochemically so that sections were sampled at 150 $\mu \mathrm{m}$ intervals. The procedures for immunohistochemical processing were identical to those described in our recent study (Jinks et al., 2002). Briefly, sections were incubated in $3 \%$ normal goat serum followed by incubation in primary c-fos antibody (1:50,000; Arnel, New York, NY) for $24-36 \mathrm{hr}$. They were then washed and exposed to a secondary biotinylated antibody and a subsequent avidin-biotin complex reaction. To visualize FLI, sections were subjected to a nickel-enhanced diaminobenzidine reaction. After coverslipping, sections were viewed under the light microscope (E-400; Nikon, Tokyo, Japan). The number of neurons expressing FLI was counted in each coronal section from C3-C6 segments. Because intradermal injections were $5 \mathrm{~mm}$ off midline, virtually all FLI was ipsilateral to the injection site, so FLI counts were restricted to the side ipsilateral to the injection. To normalize data, the 10 sections with highest counts were selected, and FLI counts were averaged for each animal. Between-group differences in FLI counts were statistically assessed by ANOVA with post hoc LSD tests. A $p$ value of $<0.05$ was considered significant. For illustrations, selected sections were imaged (Fig. 3) with a video camera attached to the microscope (DC-330; Dage MTI, Michigan City, IN) and either traced (Fig. 2) or superimposed on line drawings of cervical spinal cord sections taken from the atlas of Paxinos and Watson (1998) to plot distributions of FLI (see Figs. $4 A-E, 6 A, B$ ).

\section{Results}

\section{Scratching behavior}

Intradermal 5-HT elicited intermittent bouts of vigorous hindlimb scratching directed toward the injection site that began after a latency of several minutes and lasted $>1 \mathrm{hr}$. Figure $1, A, D$, and $G$, shows histograms of the time course of scratching bouts in three separate groups. Although there was some variability, in each case, scratching peaked within the initial 10-30 min with a second increase at 40-60 $\mathrm{min}$, confirming previous reports (Jinks and Carstens, 2002; Nojima and Carstens, 2003). Overall, there were significant between-group differences in both the number $\left(F_{(5,38)}=5.8 ; p<0.001\right)$ and duration $\left(F_{(5,38)}=4.6 ; p=\right.$ 0.002 ) of scratching bouts. Control intradermal saline injections elicited very low levels of scratching, as shown in Figure $1 B$. Indeed, intradermal saline elicited scratching in only three of eight rats (Fig. $1 \mathrm{~B}$ ), and the mean total number of scratching bouts per 60 min elicited by saline was significantly lower (LSD; $p<0.001$ ) compared with 5-HT (Fig. 1C). Similarly, the total cumulative duration of the scratching bouts over the $60 \mathrm{~min}$ observation 



Figure 1. Scratching behavior elicited by intradermal injection of 5 -HT: effects of naltrexone (NTX) and morphine (MOR). All values are means \pm SEM for six to eight rats. ${ }^{*} p<0.05$ (paired $t$ test) compared with the corresponding control (Con). A, Graph plots mean number of scratching bouts per 2 min versus time to show time course of intradermal 5-HT-evoked scratching bouts. $B$, Graph as in $A$ for scratching elicited by intradermal saline. C, Bar graph plots the mean total number of scratching bouts over the 60 min observation period elicited by intradermal 5 -HT (open bar) or saline (filled bar). D- $F$, Graphs as in $A-$ C plotting time course of scratching elicited by intradermal 5 -HT preceded by intraperitoneal saline $(D)$ or naltrexone $(E)(5 \mathrm{mg} / \mathrm{kg}$, i.p.). $F$, Graph plots the mean total number of scratching bouts for 5 -HT only and 5-HT plus naltrexone treatment groups. $G-I$, Graphs as in A-C plotting time course of scratching elicited by intradermal 5-HT alone ( $G$ ) or 5-HT preceded by morphine $(H)(3 \mathrm{mg} / \mathrm{kg}$, i.p.). I, Mean total number of scratching bouts for 5-HT only and 5-HT plus morphine treatment groups. J, Graph as in $A$ for scratching elicited by intradermal saline in animals pretreated with morphine. $K$, Graph plots mean number of scratching bouts for rats receiving intradermal saline alone or preceded by morphine $(\mathrm{NaCl}+\mathrm{MOR})$.

period was significantly greater (LSD; $p<0.01$ ) for 5 -HT (mean \pm SEM, $277.7 \pm 81.0 \mathrm{sec}$ ) compared with saline controls (mean \pm SEM, $0.8 \pm 0.5 \mathrm{sec}$ ).

After intraperitoneal administration of naltrexone, 5-HTinduced scratching was reduced (Fig. $1 E$ ) compared with 5-HTevoked scratching preceded by intraperitoneal saline throughout the observation period (Fig. 1D). The total number of 5-HTevoked scratching bouts over the 60 min observation period was significantly lower (LSD; $p<0.003$ ) after naltrexone pretreatment (Fig. $1 F$ ), as was the total cumulative duration of the scratching bouts (LSD; $p=0.007$; naltrexone, $86.8 \pm 32.8 \mathrm{sec}$; saline, $286.9 \pm 70.4 \mathrm{sec})$.

In contrast, morphine did not significantly affect 5-HTevoked scratching behavior. Figure $1 G, H$ shows that the time course of scratching was similar in the groups pretreated with saline and morphine. There was no significant difference (LSD; $p=0.772$ ) in the total number of scratching bouts between saline- and morphine-pretreated groups (Fig. $1 I$ ), nor was there a significant difference in the cumulative duration of scratching bouts (LSD; $p=0.394$; morphine, $191.6 \pm 30 \mathrm{sec}$; saline, $265.5 \pm$ $80 \mathrm{sec})$.

An additional group received intraperitoneal morphine followed by intradermal saline to verify that morphine itself did not induce scratching. During the $30 \mathrm{~min}$ period between the intraperitoneal administration of morphine and the intradermal saline injections, rats were videotaped. None $(n=8)$ exhibited any scratching during this period. After intradermal saline, there was very little scratching (Fig. $1 \mathrm{~J}$ ). Only two of the eight rats exhibited a scratching bout, and the average cumulative bout duration in this group $(0.59 \mathrm{sec})$ was slightly lower compared with the saline controls ( $0.8 \mathrm{sec}$; see above). Figure $1 \mathrm{~K}$ shows the very low mean number of scratching bouts in the saline and saline plus morphine groups ( 0.9 and 0.25 , respectively), which did not differ significantly.

\section{Fos-like immunoreactivity}

To determine which cervical spinal segments might contain neurons activated by 5-HT injections into skin at the nape of the neck, we examined horizontal sections. The top right drawing in Figure 2 shows a horizontal section through the upper dorsal horn on one side (plane of section shown to left). There was extensive FLI at the lateral edge of the dorsal horn that was more or less continuous throughout C3-C6 segments, consistent with the dermatomal innervation of the neck (Takahashi and Nakajima, 1996). The bottom left drawing in Figure 2 is a coronal section through $\mathrm{C} 5$; the dots show the distribution of FLI from an animal that received an intradermal injection of 5-HT. Figure $3 \mathrm{~A}$ shows photomicrographs of FLI (black-stained cell nuclei) in an individual midcervical section from another animal that received intradermal 5-HT, and Figure $4 C$ shows the distribution of 5-HT-evoked FLI plotted on drawings of C4-C6 sections from one animal. These examples show that FLI was distributed almost exclusively in the superficial laminas (I-III) at the far lateral edge of the dorsal horn. Because intradermal injections were off midline, virtually all FLI was ipsilateral to the injection site; a small 




Figure 2. Cervical spinal distribution of FLl evoked by intradermal 5-HT. The top left drawing is a three-dimensional view of the cervical (C2-C5) spinal cord. The gray surface indicates the plane of section for the drawing to the right, which is a horizontal section through the middorsal horn of the left side of the cervical spinal cord. In the right drawing, the dotted line to the right indicates the midline, and the thin lines indicate the lateral (left) and medial (right) margins of the spinal gray matter. Dots indicate cells expressing FLI. The bottom left drawing is an enlargement of a coronal section through C5, showing the dorsal horn laminas (dotted lines) and distribution of FLI (dots) in the lateral dorsal horn [adapted from Paxinos and Watson (1998)].

amount of FLI in a corresponding location on the contralateral side was observed in only one animal.

There was a significant between-group difference in FLI counts for the entire superficial dorsal horn $\left(F_{(4,28)}=18.75 ; p<\right.$ 0.001 ), with groups receiving intradermal $5-\mathrm{HT}$ alone or after intraperitoneal morphine or naltrexone all exhibiting significantly higher counts compared with the groups receiving intradermal saline alone or saline plus morphine (LSD; $p<0.001$ for all comparisons) (Fig. 5). Similarly, there were significantly higher FLI counts in lamina I $\left(F_{(3,20)}=6.1 ; p=0.004\right)$ and laminas II-III $\left(F_{(3,20)}=12.5 ; p<0.001\right)$ in each 5-HT treatment group compared with the group receiving intradermal saline. Figures $3 B$ and $4 A$ show that control intradermal injection of saline elicited a small amount of FLI in the lateral superficial dorsal horn, presumably because of intradermal placement of the injection needle.

Compared with intradermal 5-HT alone (Figs. 3A, 4C), there was no significant change in intradermal 5-HT-evoked FLI in the superficial dorsal horn of animals pretreated with naltrexone $(\mathrm{LSD} ; p=0.74)$ (Figs. $3 C, 4 D$, 5). Similarly, FLI elicited by 5-HT in morphine-pretreated animals was not significantly different from that evoked by $5-\mathrm{HT}$ alone (LSD; $p=0.32$ ) (Figs. 3D, 4E, 5). Morphine pretreatment resulted in a numeric reduction in FLI elicited by intradermal saline (Figs. $4 B, 5$ ); however, this reduction was not significantly different from FLI in animals receiving intradermal saline alone (LSD; $p=0.61$ ) (Fig. 5).

Intradermal capsaicin elicited FLI in the lateral cervical dorsal horn (Fig. 6A) in a distribution similar to that elicited by intradermal 5-HT. The averaged counts of FLI elicited by capsaicin (Fig. 6C) were smaller compared with 5-HT (Fig. 5), possibly because the lower capsaicin concentration $(1 \mathrm{mM})$ was less effective in exciting cutaneous nerve fibers. Importantly, pretreatment with morphine significantly attenuated capsaicin-evoked FLI (LSD; $p=0.016)($ Fig. $6 B, C)$.

\section{Discussion}

The present results confirm previous reports that intradermal 5-HT elicits scratching behavior in rats (Thomsen et al., 2001; Jinks and Carstens, 2002), and that the opiate antagonist naloxone significantly attenuates 5 -HT-induced scratching (Yamaguchi et al., 1999). Because opiate antagonists also reduce experimentally induced itch sensation (Heyer et al., 1997) and are beneficial in reducing pruritus of cholestasis and other disorders (Monroe, 1989; Bergasa et al., 1992; Peer et al., 1996), the sensitivity to naltrexone of 5-HT-induced scratching suggests that it is itch related. We also observed that morphine did not significantly affect scratching or spinal FLI elicited by intradermal 5-HT, although it did reduce capsaicin-evoked FLI, consistent with its analgesic action and its lack of antipruritic effect. Previous reports that opiates can exacerbate itch sensation in humans (Lanz et al., 1982; Stenseth et al., 1985; Ballantyne et al., 1988) would predict an increase in 5-HT-evoked scratching, which was not observed presently.

5 -HT is an inflammatory mediator associated with pain and hyperalgesia (Jensen et al., 1991; Taiwo and Levine, 1992; Abbott et al., 1996) and is liberated from rodent mast cells and platelets in many inflammatory responses (Gustafsson, 1980; Purcell et al., 1989). In humans, intradermal injection (up to $20 \mu \mathrm{g} / 20 \mu \mathrm{l}$ ) (Keele and Armstrong, 1964; Fjellner and Hägermark, 1979; Hägermark, 1995) or iontophoresis of 5-HT (1\%) (Weisshaar et al., 1997 ) is weakly pruritic. A recent study reported that $0.1 \mathrm{~mm}$ 5-HT delivered by intradermal microdialysis elicited weak itch in four subjects and weak pain in eight subjects, whereas $3 \mathrm{~mm}$ capsaicin uniformly elicited moderate pain (Schmelz et al., 2003) The present results show that both agents evoked spinal FLI, although 5-HT was more effective than capsaicin, presumably because of the difference in concentrations ( $94 \mathrm{~mm}$ 5-HT vs $1 \mathrm{~mm}$ capsaicin). In Sprague Dawley rats and ICR mice, 5-HT appears to be a more important itch mediator than histamine based on the ability to induce scratching (Kitagawa et al., 1997; Maekawa et al., 2000; Inagaki et al., 2001; Thomsen et al., 2001; Jinks and Carstens, 2002), although more studies are needed to determine whether different rat strains exhibit differential sensitivity to pruritogens, as is the case for mice (Inagaki et al., 2001). The degree of 5-HT-evoked scratching increases in a dose-related manner but is suppressed at higher doses (Yamaguchi et al., 1999), where its nociceptive effect may predominate and inhibit itch. Although these observations support the use of directed hindlimb scratching as an itch model, it should be noted that scratching per se may not invariably reflect itch. Scratching is part of the normal grooming repertoire (Van Wimersma Greidanus and Maigret, 1988), and arthritic rats exhibited scratching that was reduced by morphine (DeCastro-Costa et al., 1987), suggesting that it was pain related. Scratching is also elicited by intracranial (Königstein, 1948; Tohda et al., 1997; Johnson et al., 1999) or intrathecal microinjection of a variety of agents (Bossut et al., 1988; Frenk et al., 1988; Wilcox, 1988), although the latter was also observed in spinalized rats (Bossut et al., 1988), suggesting a direct motor effect.

Spinal FLI evoked by intradermal 5-HT was almost com- 
pletely restricted to superficial laminas in the lateral dorsal horn, similar to the distribution of cervical FLI evoked presently by capsaicin (Fig. 6A) as well as the lumbar FLI elicited by intradermal or iontophoretic application of 5-HT, capsaicin, and other algesic stimuli (histamine, nicotine, formalin, pinch) to the rat's hindpaw (Jinks et al., 2002). These findings are consistent with electrophysiological studies showing that most wide dynamic range (WDR) and nociceptive-specific type (as well as a few mechanically insensitive) neurons in lamina I respond to intradermal injection of histamine, 5-HT, capsaicin, and other agents (Jinks and Carstens, 2000, 2002). Moreover, intradermal 5-HT excited lamina I neurons over a prolonged time course that matched scratching behavior (Jinks and Carstens, 2002), providing additional support for 5-HT-responsive superficial dorsal horn neurons in the central transmission of itch in the rat. Because such neurons can signal both noxious and pruritic input, it is feasible that itch is signaled by a low firing rate and pain is signaled by a higher firing rate, the longstanding "intensity" theory of itch. In this scenario, our finding that morphine reduced capsaicinbut not 5-HT-evoked FLI might be explained by a differential suppression of nociceptive but not pruritic input onto a common neuron. This could be accomplished by the well known ability of morphine to presynaptically inhibit nociceptor afferents, although effects of morphine on itch-selective primary afferents are unknown. Another possibility is that itch is primarily signaled by a population of spinal neurons that responds selectively to 5-HT. Such neurons were rare in our previous study (Jinks and Carstens, 2002), but additional studies might uncover a larger population analogous to the mechanically insensitive lamina I spinothalamic tract neurons recently described in cats, some of which responded selectively to histamine (Andrew and Craig, 2001). Conversely, chemogenic pain would be signaled by neurons selectively receiving nociceptive but not pruritic input. Al-
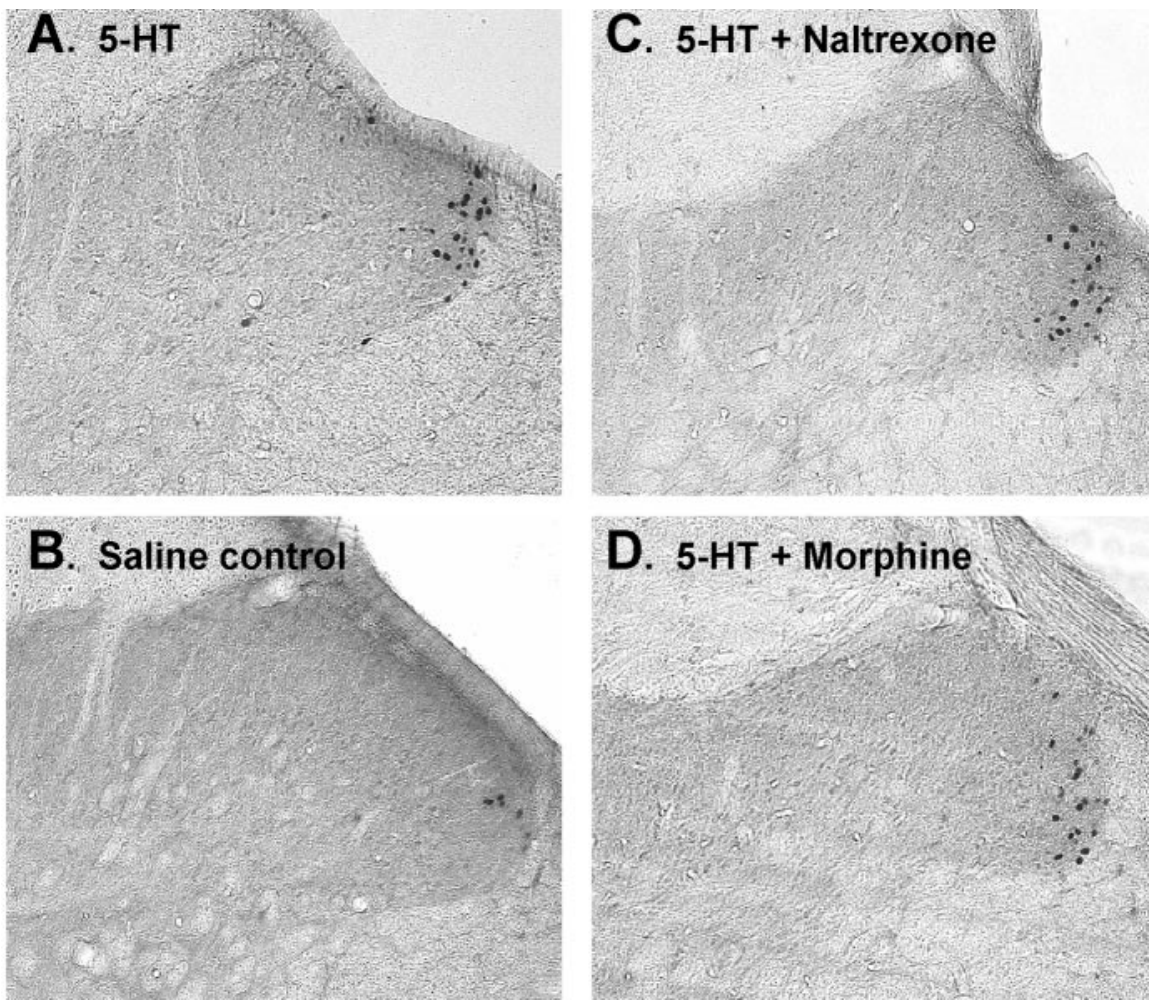

Figure 3. Photomicrographs of $\mathrm{FLI}$ in the superficial dorsal horn of the cervical spinal cord. $A$, Midcervical section from a rat receiving an intradermal injection of $5-\mathrm{HT}$ at the nape of the neck. Black dots are cell nuclei expressing FLI. B, Section from a rat receiving an intradermal injection of saline. C, Section from a rat receiving intradermal $5-\mathrm{HT}$ preceded by naltrexone $(5 \mathrm{mg} / \mathrm{kg}$, i.p.). D, Section from rat receiving intradermal 5 -HT preceded by morphine ( $3 \mathrm{mg} / \mathrm{kg}$, i.p.). Magnification, $40 X$.

though most superficial dorsal horn neurons that responded to capsaicin also responded to 5-HT, some did not (Jinks and Carstens, 2002), and one might speculate that neurons with a dominant nociceptive input are subject to morphine depression, whereas those with pruritoceptive input are not. It would be interesting to know whether neurons selectively responsive to pruritogens are resistant to or even facilitated by morphine.

Systemic administration of morphine, at a dose that inhibits withdrawal reflexes (Carstens and Ansley, 1993) and responses of nociceptive dorsal horn neurons to noxious stimuli (Carstens, 1997; Douglass and Carstens, 1997) and that presently reduced spinal FLI evoked by intradermal capsaicin (Fig. 6), did not sig-
A. $\mathrm{NaCl}$ Control



C. $5-\mathrm{HT}$
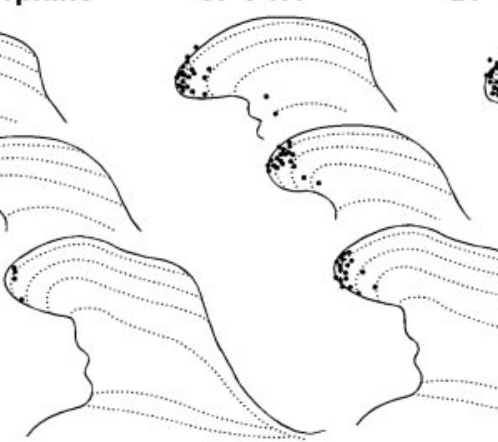

D. $\mathbf{5 - H T}+$ Naltrex.
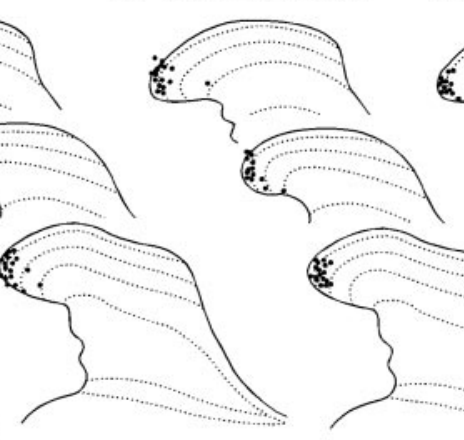

E. 5-HT + Morphine

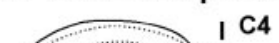



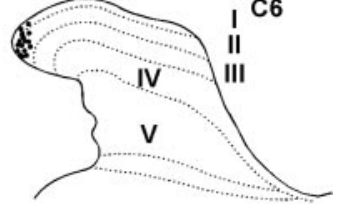

Figure 4. Distribution of FLl in the cervical dorsal horn. $A-E$, Line drawings of $C 4$ - 6 cervical segments adapted from the atlas of Paxinos and Watson (1998). Laminas are indicated by dotted lines and are labeled in $E$. Dots in each section show $\mathrm{FLI}$ transferred from a photomicrograph of the corresponding section. $A$, From an animal receiving intradermal saline only. $B$, Drawings as in $A$ from a rat receiving intradermal saline preceded by intraperitoneal morphine. C, Drawings as in $A$ from a rat receiving intradermal $5-H T$ only. D, Drawings as in $A$ from a rat receiving intradermal 5 -HT preceded by naltrexone (Naltrex.) (5 mg/kg, i.p.). E, Drawings as in $A$ from a rat receiving intradermal 5 -HT preceded by morphine ( $3 \mathrm{mg} / \mathrm{kg}$, i.p.). 




Figure 5. Counts of FLl in the superficial dorsal horn of the cervical spinal cord. The bar graph plots mean FLI count for each treatment group indicated. Shaded, open, and filled bars give mean FLI counts in lamina I (Lam. I), laminas II-III (Lam. II-III), and the entire superficial dorsal horn (Total SDH), respectively. Error bars indicate means \pm SEM for five to seven rats. ${ }^{*} p<0.05$ compared with corresponding FLI counts in saline control (CON) group. MOR, Morphine; NTX, naltrexone.

nificantly alter counts of FLI elicited by intradermal 5-HT. This result is consistent with the present lack of effect of morphine on scratching behavior elicited by intradermal 5-HT, and suggests that superficial dorsal horn neurons activated by 5-HT may primarily be involved in signaling itch rather than pain. It was reported previously that morphine reduced FLI elicited by application of histamine to rat hindpaw skin that had been abraded to disrupt the epithelial diffusion barrier (Yao et al., 1992). However, histamine does not elicit scratching in Sprague Dawley rats (Thomsen et al., 2001; Jinks and Carstens, 2002) yet strongly excites WDR and nociceptive-specific dorsal horn neurons (Carstens, 1997; Jinks and Carstens, 2000, 2002) in a morphinesensitive manner (Carstens, 1997), suggesting that histamine is algesic rather than pruritic in rats. We reported previously that intrathecal morphine at low doses $(0.1-1 \mu \mathrm{M})$ enhanced responses of some rat lamina I neurons to noxious heat and intradermal histamine while inhibiting them at a higher $(10 \mu \mathrm{M})$ dose (Jinks and Carstens, 2000). It is of interest to determine whether morphine affects lamina I neuronal responses to intradermal 5-HT; however, this may prove difficult, because successive neuronal responses to 5-HT as well as scratching exhibit marked tachyphylaxis (Jinks and Carstens, 2002).

A. Capsaicin

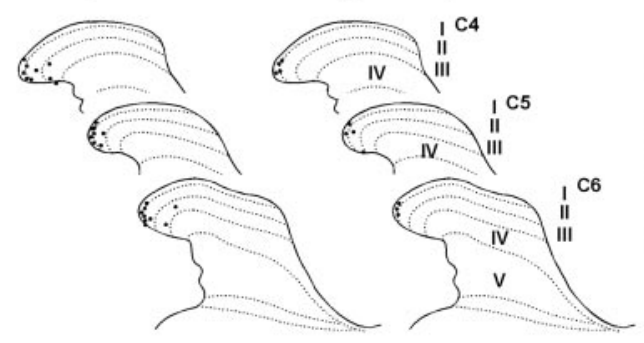

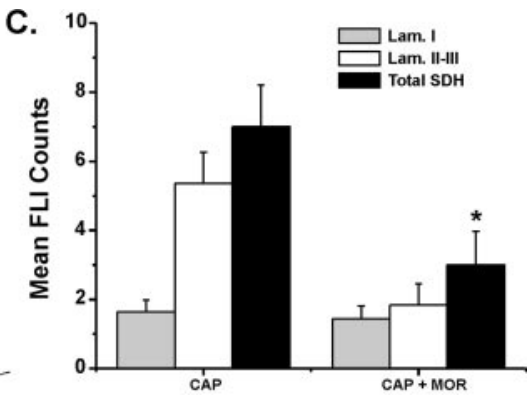

Figure 6. Capsaicin-evoked FLI and reduction by morphine. $A$, Line drawings as in Figure 4 showing distribution of FLI from a rat receiving intradermal capsaicin. $B$, Drawing as in $A$ from a rat receiving intradermal capsaicin (Cap.) preceded by intraperitoneal morphine. C, Bar graph of FLI counts in lamina I (Lam. I), laminas II-III (Lam. II-III), and the entire superficial dorsal horn (Total SDH) for groups receiving intradermal capsaicin (CAP) and capsaicin preceded by intraperitoneal morphine (CAP + MOR). Format is as in Figure $5 .{ }^{*} p<0.05$, significant difference from CAP group.
Although naltrexone significantly attenuated scratching behavior, it did not affect counts of FLI evoked by intradermal 5-HT. This result was surprising, because epidural administration of opioid analgesics such as fentanyl often induces pruritus (Gurkan and Toker, 2002) in support of a spinal opioid action on itch-signaling pathways. Nevertheless, it is conceivable that naltrexone suppresses the itch-related scratching behavior at a supraspinal rather than spinal site. This interpretation is supported by recent data showing that intracisternal but not intrathecal (lumbosacral) administration of naloxone attenuated itchrelated hindlimb biting behavior elicited by intraplantar 5-HT in mice (Hagiwara et al., 1999; Yageta et al., 2000). Intracerebroventricular (Königstein, 1948; Tohda et al., 1997) and intramedullary microinjection of opiates (Thomas et al., 1993) induces marked facial scratching that is reversed by the opioid antagonists naloxone or naltrexone. It is therefore conceivable that the ability of naltrexone to attenuate scratching behavior was attributable to blockade of the transmission of itch signals at a medullary (and/or other supraspinal) level. If so, future studies will hopefully shed light on the neural mechanisms by which supraspinal itch signals are translated into motor commands to initiate antipruritic scratching behavior.

\section{References}

Abbott FV, Hong Y, Blier P (1996) Activation of 5- $\mathrm{HT}_{2 \mathrm{~A}}$ receptors potentiates pain produced by inflammatory mediators. Neuropharmacology 35:99-110.

Andrew D, Craig AD (2001) Spinothalamic lamina 1 neurons selectively sensitive to histamine: a central neural pathway for itch. Nat Neurosci 4:72-77.

Ballantyne JC, Loach AB, Carr DB (1988) Itching after epidural and spinal opiates. Pain 33:149-160.

Bergasa NV, Talbot TL, Alling DW, Schmitt JM, Walker EC, Baker BL, Korenman JC, Park Y, Hoofnagle JH, Jones EA (1992) A controlled trial of naloxone infusions for the pruritus of chronic cholestasis. Gastroenterology 102:544-549.

Bossut D, Frenk H, Mayer DJ (1988) Is substance P a primary afferent neurotransmitter for nociceptive input? II. Spinalization does not reduce and intrathecal morphine potentiates behavioral responses to substance P. Brain Res 445:232-239.

Carstens E (1997) Responses of rat spinal dorsal horn neurons to intracutaneous microinjection of histamine, capsaicin, and other irritants. J Neurophysiol 77:2499-2514.

Carstens E, Ansley D (1993) Hindlimb flexion withdrawal evoked by noxious heat in conscious rats: magnitude measurement of stimulusresponse function, suppression by morphine and habituation. J Neurophysiol 70:621-629.

DeCastro-Costa M, Gybels J, Kupers R, Van Hees J (1987) Scratching behaviour in arthritic rats: a sign of chronic pain or itch? Pain 29:123-131.

Doi-Saika M, Tokunaga A, Senba E (1997) Intradermal 5-HT induces Fos expression in rat dorsal horn neurons not via 5 -HT3 but via 5-HT2A receptors. Neurosci Res 29:143-149.

Douglass DK, Carstens E (1997) Responses of rat sacral spinal neurons to mechanical and noxious thermal stimulation of the tail. J Neurophysiol 77:611-620.

Fjellner B, Hägermark O (1979) Pruritus in polycythemia vera: treatment with aspirin and possibility of platelet involvement. Acta Derm Venereol 59:505-512.

Frenk H, Bossut D, Urca G, Mayer DJ (1988) Is substance $\mathrm{P}$ a primary afferent neurotransmitter for nociceptive input? I. Analysis of painrelated behaviors resulting from intrathecal administration of substance $P$ and 6 excitatory compounds. Brain Res 455:223-231. 
Gurkan Y, Toker K (2002) Prophylactic ondansetron reduces the incidence of intrathecal fentanyl-induced pruritus. Anesth Analg 95:1763-1766.

Gustafsson B (1980) Cytofluorometric analysis of anaphylactic secretion of 5-hydroxytryptamine and heparin from rat mast cells. Int Arch Allergy Appl Immunol 63:121-128.

Hägermark O (1995) Itch mediators. Semin Dermatol 14:271-276.

Hagiwara K, Nojima H, Kuraishi Y (1999) Serotonin-induced biting of the hind paw is itch-related response in mice. Pain Res 14:53-59.

Heyer G, Dotzer M, Diepgen TL, Handwerker HO (1997) Opiate and H1 antagonist effects on histamine induced pruritus and alloknesis. Pain 73:239-243.

Inagaki N, Nagao M, Igeta K, Kawasaki H, Kim JF, Nagai H (2001) Scratching behavior in various strains of mice. Skin Pharmacol Appl Skin Physiol 14:87-96.

Jensen K, Tuxen C, Pedersen-Bjergaard U, Jansen I (1991) Pain, tenderness, wheal and flare induced by substance-P, bradykinin and 5-hydroxytryptamine in humans. Cephalagia 11:175-182.

Jinks SL, Carstens E (2000) Superficial dorsal horn neurons identified by intracutaneous histamine: chemonociceptive responses and modulation by morphine. J Neurophysiol 84:616-627.

Jinks SL, Carstens E (2002) Responses of superficial dorsal horn neurons to intradermal serotonin and other irritants: comparison with scratching behavior. J Neurophysiol 87:1280-1289.

Jinks SL, Simons CT, Dessirier JM, Carstens MI, Antognini JF, Carstens E (2002) C-fos induction in rat superficial dorsal horn following cutaneous application of noxious chemical or mechanical stimuli. Exp Brain Res 145:261-269.

Johnson MD, Ko M, Choo KS, Traynor JR, Mosberg HI, Naughton NN, Woods JH (1999) The effects of the phyllolitorin analogue [desTrp(3), Leu(8)] phyllolitorin on scratching induced by bombesin and related peptides in rats. Brain Res 839:194-198.

Jones EA, Bergasa NV (2000) Evolving concepts of the pathogenesis and treatment of the pruritus of cholestasis. Can J Gastroenterol 14:33-40.

Keele CA, Armstrong D (1964) Substances producing pain and itch. Baltimore: Lippincott, Williams, and Wilkins.

Kitagawa K, Andoh T, Nagasawa T, Satoh M, Kuraishi Y (1997) Different effects of antagonists to histamine and serotonin receptors on itch-related behavior induced by substance P, histamine and serotonin in mice. Jpn J Pharmacol 73 [Suppl I]:93.

Königstein H (1948) Experimental study of itch stimuli in animals. AMA Arch Dermatol Syphilol 57:829-849.

Kuraishi Y, Nagasawa T, Hayashi K, Satoh M (1995) Scratching behavior induced by pruritogenic but not algesiogenic agents in mice. Eur J Pharmacol 275:229-233.

Lanz E, Theiss D, Riess W, Sommer U (1982) Epidural morphine for postoperative analgesia: a double-blind study. Anesth Analg 61:236-240.

Luo C, Chen J, Li HL, Li JS (1998) Spatial and temporal expression of c-Fos protein in the spinal cord of anesthetized rat induced by subcutaneous bee venom injection. Brain Res 806:175-185.

Maekawa T, Nojima H, Kuraishi Y (2000) Itch-associated responses of afferent nerve innervating the murine skin: different effects of histamine and serotonin in ICR and ddY mice. Jpn J Pharmacol 84:462-466.

Monroe EW (1989) Efficacy and safety of nalmefen in patients with severe pruritus caused by chronic urticaria and atopic dermatitis. J Am Acad Dermatol 21:135-136.

Nojima H, Carstens E (2003) Quantitative assessment of directed hind limb scratching behavior as a rodent itch model. J Neurosci Methods 126:137-143.

Paxinos G, Watson C (1998) The rat brain in stereotaxic coordinates, Ed 4. New York: Academic.

Peer G, Kivity S, Agami O, Fireman E, Silverberg D, Blum M, Laina A (1996) Randomised cross-over trial of naltrexone in uremic pruritus. Lancet 348:1552-1554.
Presley RW, Menetrey D, Levine JD, Basbaum AI (1990) Systemic morphine suppresses noxious stimulus-evoked Fos protein-like immunoreactivity in the rat spinal cord. J Neurosci 10:323-335.

Purcell WM, Cohen DL, Hanahoe TH (1989) Comparison of histamine and 5-hydroxytryptamine content and secretion in rat mast cells isolated from different anatomical locations. Int Arch Allergy Appl Immunol 90:382-386.

Schmelz M, Schmidt R, Bickel A, Handwerker HO, Torebjork HE (1997) Specific C-receptors for itch in human skin. J Neurosci 17:8003-8008.

Schmelz M, Schmidt R, Weidner C, Hilliges M, Torebjork HE, Handwerker HO (2003) Chemical response pattern of different classes of C-nociceptors to pruritogens and algogens. J Neurophysiol 89:2441-2448.

Schmidt R, Torebjork E, Jorum E (1993) Pain and itch from intraneural microstimulation. In: Proceedings of the Seventh World Congress on Pain. Progress in Pain Research and Management, Vol 2 (Gebhart GF, Hammond DL, Jensen TS, eds), p 143. Seattle: International Association for the Study of Pain.

Stenseth R, Sellevold O, Breivik H (1985) Epidural morphine for postoperative pain: experience with 1085 patients. Acta Anaesthesiol Scand 29:148-156.

Taiwo YO, Levine JD (1992) Serotonin is a directly acting hyperalgesic agent in the rat. Neuroscience 48:485-490.

Takahashi Y, Nakajima Y (1996) Dermatomes in the rat limbs as determined by antidromic stimulation of sensory C-fibers in spinal nerves. Pain 67:197-202.

Thomas DA, Williams GM, Iwata K, Kenshalo Jr DR, Dubner R (1993) Multiple effects of morphine on facial scratching in monkeys. Anesth Analg 77:933-935.

Thomsen JS, Petersen MB, Benfeldt E, Jensen SB, Serup J (2001) Scratch induction in the rat by intradermal serotonin: a model for pruritus. Acta Derm Venereol 81:250-254.

Tohda C, Yamaguchi T, Kuraishi Y (1997) Intracisternal injection of opioids induces itch-associated response through mu-opioid receptors in mice. Jpn J Pharmacol 74:77-82.

Tuckett RP (1982) Itch evoked by electrical stimulation of the skin. J Invest Dermatol 79:368-373.

Van Wimersma Greidanus TB, Maigret C (1988) Grooming behavior induced by substance P. Eur J Pharmacol 154:217-220.

Wahlgren CF (1991) Itch and atopic dermatitis: clinical and experimental studies. Acta Derm Venereol Suppl (Stockh) 165:1-53.

Ward L, Wright E, McMahon SB (1996) A comparison of the effects of noxious and non-noxious counterstimuli on experimentally induced itch and pain. Pain 64:129-138.

Weisshaar E, Ziethen B, Gollnick H (1997) Can a serotonin type 3 (5-HT3) receptor antagonist reduce experimentally induced itch? Inflamm Res 46:412-416.

Wilcox GL (1988) Pharmacological studies of grooming and scratching behavior elicited by spinal substance $P$ and excitatory amino acids. Ann NY Acad Sci 525:228-236.

Woodward DF, Nieves AL, Spada CS, Williams LS, Tuckett RP (1995) Characterization of a behavioral model for peripherally evoked itch suggests platelet-activating factor as a potent pruritogen. J Pharmacol Exp Ther 272:758-765.

Yageta Y, Nojima H, Kuraishi Y (2000) Involvement of intracerebral opioid receptors in itch-associated behavioral responses of mice. 21st Japanese Narcotic Research Conference, Nagasaki, Japan, August, Abstr 90-92.

Yamaguchi T, Nagasawa T, Satoh M, Kuraishi Y (1999) Itch-associated response induced by intradermal serotonin through $5-\mathrm{HT}_{2}$ receptors in mice. Neurosci Res 35:77-83.

Yao GL, Tohyama M, Senba E (1992) Histamine-caused itch induces Foslike immunoreactivity in dorsal horn neurons: effect of morphine pretreatment. Brain Res 599:333-337. 